Pacific Journal of Mathematics

EXTENSION THEOREMS FOR FUNCTIONS OF VANISHING 


\title{
EXTENSION THEOREMS FOR FUNCTIONS OF VANISHING MEAN OSCILLATION
}

\section{Peter J. Holden}

\begin{abstract}
A locally integrable function is said to be of vanishing mean oscillation (VMO) if its mean oscillation over cubes in $\mathbf{R}^{d}$ converges to zero with the volume of the cubes. We establish necessary and sufficient conditions for a locally integrable function defined on a bounded measurable set of positive measure to be the restriction to that set of a VMO function.
\end{abstract}

1. Introduction. Let $F$ be a locally integrable function on $\mathbf{R}^{d}$ and let $Q$ be a cube in $\mathbf{R}^{d}$ with sides parallel to the axes. (We denote the set of all such cubes in $\mathbf{R}^{d}$ by $\mathfrak{F}^{\prime}$.) We denote the Lebesgue measure of $Q$ by $|Q|$ and the length of $Q$ by $l(Q)$. We denote the average of $F$ on $Q$ by $F_{Q}$; that is $F_{Q}=\frac{1}{|Q|} \int_{Q} F d t$. We say $F$ is of bounded mean oscillation (abbreviated $\mathrm{BMO}\left(\mathbf{R}^{d}\right)$ or simply $\mathrm{BMO}$ ) if

$$
\sup _{Q \in \mathfrak{F}^{\prime}} \frac{1}{|Q|} \int_{Q}\left|F-F_{Q}\right|<\infty \text {. }
$$

We denote this supremum by $\|F\|_{*}$. \|\|$_{*}$ defines a norm on BMO and $\mathrm{BMO}$ is a Banach space with respect to this norm. (We identify functions which differ by a constant.) If in (1.1) we restrict the cubes to be dyadic we obtain the space dyadic-BMO and we denote the corresponding norm by \|\|$_{*, d}$. (By a dyadic cube we mean a cube of the form $Q=\left\{k_{j}<x_{j}<\left(k_{j}+1\right) 2^{-n} ; 1 \leq j \leq d\right\}$ where $n$ and $k_{j}, 1 \leq j \leq d$, are integers.) We will denote the set of dyadic cubes of length $2^{-n}$ by $D_{n}$ and $Q_{0}$ will denote the dyadic unit cube. The function space BMO was introduced in 1961 by John and Nirenberg [7] who proved the following fundamental theorem:

THEOREM 1.1. Let $F$ be a locally integrable function on $\mathbf{R}^{d}$, and for each $n \in \mathbf{Z}$ define:

Then,

$$
\bar{\mu}_{n}(F)=\inf \left\{\frac{1}{\lambda}: \sup _{l(Q) \leq 2^{-n}} \inf _{a \in \mathbf{R}} \frac{1}{|Q|} \int_{Q} e^{\lambda|F-a|}<2\right\} .
$$

(1) $F \in \mathrm{BMO}$ if and only if (2) $\sup _{n \in \mathbf{Z}} \bar{\mu}_{n}(F)<\infty$. 
The implication $(2) \Rightarrow(1)$ is straightforward while $(1) \Rightarrow(2)$ is obtained by means of a Calderon-Zygmund stopping time argument. (This result and other basic results on BMO can be found in [4] and [12].)

A closed subspace of BMO that we will be mainly concerned with, is the space of functions of vanishing mean oscillation (VMO) which was introduced by Sarason in [11] and is defined as:

$$
\mathrm{VMO}=\left\{F \in \mathrm{BMO}: \lim _{\delta \rightarrow 0}\left(\sup _{\substack{Q \in \mathfrak{F}^{\prime} \\ l(Q)<\delta}} \frac{1}{|Q|} \int_{Q}\left|F-F_{Q}\right|\right)=0\right\}
$$

Equivalently, by the theorem of John and Nirenberg, $F \in \mathrm{VMO}$ if and only if $F \in \mathrm{BMO}$ and $\lim _{n \rightarrow \infty} \bar{\mu}_{n}(F)=0$.

If $E$ is a Lebesgue measurable subset of $\mathbf{R}^{d}$ of positive measure (throughout we will always assume $E$ has positive measure), we can ask for necessary and sufficient conditions for a locally integrable function defined on $E$ to be the restriction to $E$ of a function in $\operatorname{BMO}\left(\mathbf{R}^{d}\right)$. This characterization was given by Wolff [15] and is based upon a technique due to Rubio de Francia [10] which generalizes Jones' factorization theorem for $A_{p}$-weights [8]. The main result of this paper is to obtain a similar characterization for VMO functions and this is the content of the following theorem:

THEOREM I. Let $E$ be a bounded measurable subset of $\mathbf{R}^{d}$ and let $f$ be a locally integrable function defined on $E$. For each $n \in \mathbf{Z}$ define:

$$
\mu_{n}(f)=\inf \left\{\frac{1}{\lambda}: \sup _{l(Q) \leq 2^{-n}} \inf _{a \in \mathbf{R}} \frac{1}{|Q|} \int_{Q \cap E} e^{\lambda|f-a|}<2\right\}
$$

Then the following are equivalent:

(1) $f$ is the restriction of a VMO function on $\mathbf{R}^{d}$ to $E$

(2) $\sup _{n \in \mathbf{Z}} \mu_{n}(f)<\infty$ and $\lim _{n \rightarrow \infty} \mu_{n}(f)=0$.

The proof of this theorem consists of two parts. In the first part we obtain a dyadic-VMO extension of $f$. We then obtain a dyadicVMO extension for each translation of $f$ and $E$ and the second part of the proof consists of averaging these extensions to obtain a VMO extension of $f$. 
Throughout $C$ will denote a positive constant which will be independent of the variables in the equation in which it occurs but which may be different at each occurrence.

2. Preliminary Results. Let $E$ be a measurable subset of $\mathbf{R}^{d}$ and let $\mathfrak{F}$ be a collection of cubes in $\mathbf{R}^{d}$ with $E \subseteq \bigcup\{Q: Q \in \mathfrak{F}\}$.

Definition. (1) if $F$ is a locally integrable function on $\mathbf{R}^{d}$, we define the maximal function of $F$ relative to $\mathfrak{F}$ by

$$
\left(M_{\mathfrak{F}} F\right)(x)=\sup _{\substack{x \in Q \\ Q \in \mathfrak{F}}} \frac{1}{|Q|} \int_{Q} F d t \quad \text { for all } x \in \bigcup\{Q: Q \in \mathfrak{F}\} .
$$

If $\mathfrak{F}=\mathfrak{F}^{\prime}$, this is the usual Hardy-Littlewood maximal function.

(2) If $f$ is a locally integrable function on $E$, we define the maximal function of $f$ relative to $\mathfrak{F}$ by

$$
\left(m_{\mathfrak{F}} f\right)(x)=\sup _{\substack{x \in Q \\ Q \in \mathfrak{F}}} \frac{1}{|Q|} \int_{Q \cap E} f d t \quad \text { for all } x \in E .
$$

DEFINITION. (1) Let $w$ be a positive locally integrable function on $E$ and let $1<p<\infty$. We say $w$ is an $A_{p}(E)$-weight relative to $\mathfrak{F}$ if

$$
\sup _{Q \in \mathfrak{F}}\left(\frac{1}{|Q|} \int_{Q \cap E} w d t\right)\left(\frac{1}{|Q|} \int_{Q \cap E}\left(\frac{1}{w}\right)^{1 /(p-1)} d t\right)^{p-1}<\infty
$$

and we denote the collection of all such weights by $A_{p}(E, \mathfrak{F})$. If $E=\mathbf{R}^{d}$ and $\mathfrak{F}=\mathfrak{F}^{\prime}$ we abbreviate $A_{p}(E, \mathfrak{F})$ by $A_{p}$ and say $w$ is an $A_{p}$-weight.

(2) We say a positive locally integrable function $w$ is an $A_{1}(E)$ weight relative to $\mathfrak{F}$ if

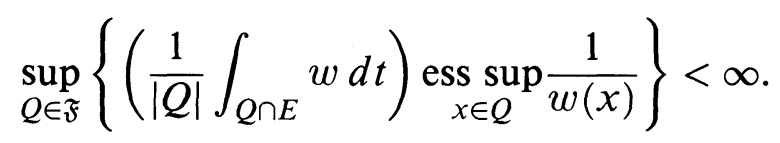

We denote the collection of all such weights by $A_{1}(E, \mathfrak{F})$.

We record some properties of $A_{p}(E, \mathfrak{F})$-weights in the following proposition.

Proposition 2.1.

(i) If $w \in A_{p}(E, \mathfrak{F})$ then $w \in A_{r}(E, \mathfrak{F})$ for all $r>p$ and $\left(\frac{1}{w}\right)^{1 /(p-1)} \in$ $A_{p /(p-1)}$.

(ii) If $w_{1}, w_{2} \in A_{1}(E, \mathfrak{F})$ then $w_{1}, w_{2}^{1-p} \in A_{p}(E, \mathfrak{F})$ for all $1<p<$ $\infty$. 
(iii) If $w \in A_{p}$ then $F=\log w \in \mathrm{BMO}$. By the theorem of John and Nirenberg (Theorem 1.1), if $F \in \mathrm{BMO}$ there exists $\delta>0$ such that $e^{\delta F} \in A_{p}$.

(iv) We mention here the following result of Coifman and Rochberg [3]: If $F \in L_{1}^{\log }\left(\mathbf{R}^{d}\right)$ and $M_{\mathfrak{F}^{\prime}} f(x)<\infty$ a.e., then for each $0<\delta<1$, $\left(M_{\mathfrak{F}^{\prime}} f\right)^{\delta} \in A_{1}$.

Definition. Let $1<p<\infty$ and let $w \in A_{p}(E, \mathfrak{F})$. We say $w$ satisfies a reverse Hölder inequality if there exists $\varepsilon>0$ such that $w^{1+\varepsilon} \in A_{p}(E, \mathfrak{F})$.

REMARK. If $w \in A_{p}$ then $w$ satisfies a reverse Hölder inequality with $\varepsilon$ depending on $p$ and supremum in (2.1). This fact may be deduced from (2.1) by a repeated application of a Calderon-Zygmund stopping time argument. See [1], [9].

The next theorem is a variation of a theorem of Muckenhoupt [9]. The proof is the same and so will be omitted.

TheOREM 2.1. Let $1<p<\infty$ and let $w \in A_{p}(E, \mathfrak{F})$ where $\mathfrak{F}$ is a collection of dyadic cubes or $\mathfrak{F}=\mathfrak{F}^{\prime}$. If $w$ satisfies a reverse Hölder inequality then there exists a constant $C>0$ such that

$$
\int_{E}\left\{m_{\mathfrak{F}}(f)\right\}^{p} w d x \leq C \int_{E}|f|^{p} w d x
$$

and

$$
\int_{E}\left\{m_{\mathfrak{F}}(f)\right\}^{q}\left(\frac{1}{w}\right)^{q / p} d x \leq C \int_{E}|f|^{q}\left(\frac{1}{w}\right)^{q / p} w d x
$$

where $q=p /(p-1)$.

By a theorem of Rubio de Francia [10], (2.2) and (2.3) imply that there exist $w_{1}, w_{2} \in A_{1}(E, \mathfrak{F})$ such that $w=w_{1} w_{2}^{1-p}$. We summarize what we need from the above in the following corollary.

CoRollary 2.1. If $w \in A_{2}(E, \mathfrak{F})$ and $w$ satisfies a reverse Hölder inequality then there exist $w_{1}, w_{2} \in A_{2}(E, \mathfrak{F})$ such that $w=w_{1} / w_{2}$.

We are now in a position to give the BMO extension theorem of Wolff [15]. 
THEOREM 2.2. If $f$ is measurable on $E$, then the following are equivalent:

(1) $f$ is the restriction of a BMO function on $\mathbf{R}^{d}$ to $E$;

(2) $\exists \lambda>0$ such that

$$
\sup _{Q \in \mathfrak{F}^{\prime}} \frac{1}{|Q|} \int_{Q \cap E} e^{\lambda\left|f-(1 /|Q \cap E|) \int_{Q \cap E} f\right|}<\infty
$$

(3) $\exists \lambda>0$ such that

$$
\sup _{Q \in \mathfrak{F}^{\prime}} \inf _{a \in \mathbf{R}} \frac{1}{|Q|} \int_{Q \cap E} e^{\lambda|f-a|}<\infty .
$$

We give the proof as it provides one of the basic steps needed in proving Theorem I.

Proof. The equivalence of (2) and (3) are straightforward and the implication $(1) \Rightarrow(3)$ is similar to the proof of $(1) \Rightarrow(2)$ in Theorem I which we give in $\S 3$.

(2) $\Rightarrow(1)$ : (2) implies that $w=e^{\lambda f / 2} \in A_{2}\left(E, \mathfrak{F}^{\prime}\right)$ and satisfies a reverse Hölder inequality. Hence by Corollary 2.1 , there exist $w_{1}, w_{2} \in$ $A_{1}\left(E, \mathfrak{F}^{\prime}\right)$ such that $w=e^{\lambda f / 2}=w_{1} / w_{2}$. Define $W_{i}=M_{\mathfrak{F}^{\prime}}\left(\chi_{E} w_{i}\right)^{1 / 2}$, $i=1$, 2. By Proposition 2.1 (iv), $W_{1}, W_{2} \in A_{1}$, i.e. $\exists C>0$ such that $W_{i} \leq M_{\mathfrak{F}^{\prime}}\left(W_{i}\right) \leq C W_{i}, i=1,2$. Since $M_{\mathfrak{F}^{\prime}}\left(\chi_{E} w_{i}\right)=m_{\mathfrak{F}^{\prime}}\left(w_{i}\right)$, a.e. on $E,(i=1,2)$, it follows that $\exists g \in L_{\infty}\left(\mathbf{R}^{d}\right), g>0$, such that

$$
g\left(\frac{W_{1}}{W_{2}}\right)^{2}=\frac{w_{1}}{w_{2}}=e^{\lambda f / 2} \quad \text { a.e. on } E \text {. }
$$

Define $F=\frac{2}{\lambda}\left\{\log g+2 \log \left(W_{1} / W_{2}\right)\right\}$. Then $F=f$ a.e. on $E$ and by Proposition 2.1 (ii) and (iii), $F \in \mathrm{BMO}\left(\mathbf{R}^{d}\right)$.

Finally we prove 2 lemmas which are needed in the next section. The first is a variation of the theorem of Coifman and Rochberg mentioned above while the second is based on Lemma 2.2 in [5].

Lemma 2.1. Let $n \in \mathbf{N}$ and let $\mathfrak{F}=\left\{Q: Q\right.$ dyadic, $Q \subseteq Q_{0}, l(Q) \geq$ $\left.2^{-n}\right\}$. Let $g \in L_{1}^{\operatorname{loc}}\left(Q_{0}\right)$ and suppose $\left(M_{\mathfrak{F}} g\right)(x)<\infty$ a.e. Then for all $0<\delta<1$, there exists $C_{\delta}>0$, depending only on $\delta$ and the dimension, such that

$$
M_{\mathfrak{F}}\left(\left(M_{\mathfrak{F}} g\right)^{\delta}\right)(x) \leq C_{\delta}\left(M_{\mathfrak{F}} g\right)^{\delta}(x)
$$


Proof. It suffices to show $\exists C_{\delta}>0$ such that $\forall Q \in \mathfrak{F}$,

$$
\frac{1}{|Q|} \int_{Q}\left(M_{\mathfrak{F}} g\right)^{\delta} d t \leq C_{\delta} \inf _{x \in Q}\left(M_{\mathfrak{F}} g\right)^{\delta}(x) \text {. }
$$

This result is established by Coifman and Rochberg in [3] for the usual Hardy-Littlewood maximal function. The proof of (2.4) for $Q \in \mathfrak{F}$ with $l(Q)<\frac{1}{2}$ is essentially the same; the relevant property of such cubes is the following:

$\exists Q_{1} \in \mathfrak{F}, l\left(Q_{1}\right)=2 l(Q)$ such that $Q \subseteq Q_{1}$ and whenever $Q_{2} \in \mathfrak{F}$ satisfies $\left|Q_{2} \cap Q\right|>0$ and $\left|Q_{2} \cap Q_{1}^{c}\right|>0$ we have $Q \subseteq Q_{2}$.

For $Q \in \mathfrak{F}$ with $l(Q) \geq \frac{1}{2},(2.4)$ follows by integrating the weak-type estimate for the Hardy-Littlewood maximal function.

LEMMA 2.2. Let $E$ be a measurable subset of the unit cube $Q_{0}$ with $0<|E|<1$. Then if $0<\beta<\log 1 /|E|, \exists H \in$ dyadic- $\operatorname{VMO}\left(Q_{0}\right)$, $\|H\|_{*, d} \leq C$ such that:

(1) $0 \leq H \leq \beta, \operatorname{supp}(H) \subseteq Q_{0}, H=\beta$ on $E$,

(2) $\sup _{Q: l(Q) \geq 1} \frac{1}{|Q|} \int_{Q} H d t \leq C$.

Proof. This is a version of Lemma 2.2 in [5] where $H$ is constructed in $\operatorname{BMO}\left(Q_{0}\right)$ satisfying (1) and (2). The argument below is the dyadic version of this construction. (See also the proof of Lemma 1.2 in [14] where a similar result is obtained.) Without loss of generality we may assume $|E| \leq 2^{-4 d}$ (otherwise we may take $H$ to be constant). Let $\left\{Q_{i}\right\}_{i \geq 1}$ be the maximal subcubes of $Q$ for which $\left|Q_{i} \cap E\right|>\frac{1}{2}\left|Q_{i}\right|$. Let $n_{0}=1$ and for each $j \geq 1$ choose $n_{j}$ so that

$$
\left|\log \sum_{i \geq n_{j}}\right| Q_{i}||>2^{j}|\log | \bigcup Q_{i}||
$$

and define $G^{(j)}=\left\{Q_{i}: n_{j} \leq i<n_{j+1}\right\}$ so that $\sum_{Q \in G^{(j)}}|Q| \leq 4^{-\beta_{j} d}\left|Q_{0}\right|$ where $\beta_{j}=2^{j} \beta_{0}, \beta_{0}=\left[\frac{1}{2 d}|\log | \cup Q_{i}||\right]$ and [ ] denotes the greatest integer function.

For each $j$ we now construct a sequence of generation $\left\{G_{i}^{(j)}\right\}_{i=1}^{\beta_{j}}$ as follows:

(1) $\operatorname{Set} G_{1}^{(J)}=G^{(j)}$.

(2) Suppose $G_{i}^{(j)}$ has been defined. For each $Q \in G_{l}^{(j)}$ let $G^{(k)}$ denote that dyadic cube of length $2^{k} l(Q)$ containing $Q$. Choose $k$ minimal so that

$$
\sum\left\{\left|Q_{i}\right|: Q_{i} \in G_{i}^{(j)}, Q_{i} \subseteq Q^{(k)}\right\}<2^{-d}\left|Q^{(k)}\right| .
$$


We define $G_{i+1}^{(j)}$ to be the maximal cubes in $\left\{Q_{r}^{(k)}: Q_{r} \in G_{i}^{(j)}\right\}$. We note that

$$
\sum\left\{|Q|: Q \in G_{\beta_{J-l}}^{(j)}\right\} \leq 2^{-d} \sum\left\{|Q|: Q \in G_{\beta_{j-l-1}}^{(j)}\right\}
$$

and hence if $Q$ is any dyadic cube then

$$
\sum_{i=1}^{\beta_{J}} \sum_{\substack{Q_{k} \in G_{l}^{(J)} \\ Q_{k} \subseteq Q}}\left|Q_{k}\right| \leq C|Q| .
$$

Hence by an argument similar to the proof of Lemma 2.1 in [5] we see that the function

$$
a_{j}=\sum_{i=1}^{\beta_{J}} \sum_{Q_{k} \in G_{t}^{(J)}} \chi_{Q}
$$

belongs to dyadic-BMO $\left(Q_{0}\right)$ with $\left\|a_{j}\right\|_{*, d} \leq C$. Furthermore $a_{j}=\beta_{j}$ on $\bigcup\left\{Q_{k}: Q_{k} \in G_{1}^{(j)}\right\}$ and since $a_{j}$ is constant on small dyadic cubes, we have in fact that $a_{j} \in$ dyadic- $\operatorname{VMO}\left(Q_{0}\right)$. We now define

$$
H=\frac{\beta}{\beta_{0}} \min \left(\sum_{j \geq 0} \frac{a_{j}}{2^{j}}, \beta_{0}\right) .
$$

REMARK. We note that Lemma 2.2 may be established without using the above construction. Indeed, it is not hard to see that the function

$$
f= \begin{cases}\beta_{j} & \text { on } \bigcup\left\{Q: Q \in G_{1}^{(j)}\right\}, \\ 0 & \text { on } Q_{0}^{c}\end{cases}
$$

satisfies the hypothesis (3) of Theorem 2.2 with

$$
E=\bigcup\left\{Q: Q \in G_{1}^{(j)}\right\} \cup Q_{0}^{c}
$$

and with $\mathfrak{F}^{\prime}$ replaced by those dyadic cubes of length $\geq \min \{l(Q)$ : $\left.Q \in G_{1}^{(j)}\right\}$ and so can be extended to a dyadic-BMO function $F$ with $\|F\|_{*, d} \leq C$ and which is constant on small dyadic cubes. This will become clear after reading $\S 3(\mathrm{i})$ below.

3. Proof of Theorem I. If $Q$ is a cube and $r>0$ we will denote by $r Q$ the cube with the same center as $Q$ and of length $r l(Q)$.

Without loss of generality we will assume $E$ is contained in $\frac{1}{3} Q_{0}$ where $Q_{0}$ is the unit cube in $\mathbf{R}^{d}$. 
$(1) \Rightarrow(2)$ : Let $F \in \mathrm{VMO}$ with $F \chi_{E}=f$ and for each $n \in \mathbf{Z}$ define

$$
\begin{aligned}
& \bar{\mu}_{n}(F)=\inf \left\{\frac{1}{\lambda}: \sup _{l(Q) \leq 2^{-n}} \inf _{a \in \mathbf{R}} \frac{1}{|Q|} \int_{Q} e^{\lambda|F-a|}<2\right\}, \\
& \bar{\mu}_{n}^{*}(F)=\inf \left\{\frac{1}{\lambda}: \sup _{l(Q) \leq 2^{-n}} \frac{1}{|Q|} \int_{Q} e^{\lambda\left|F-F_{Q}\right|}<2\right\}, \\
& \|F\|_{*, n}=\sup _{l(Q) \leq 2^{-n}} \frac{1}{|Q|} \int_{Q}\left|F-F_{Q}\right| .
\end{aligned}
$$

Since $F \in \mathrm{BMO}, \exists C>0$ such that $\forall n \geq 0$,

$$
\|F\|_{*, n} \leq C \text { and } \quad \lim _{n \rightarrow \infty}\|F\|_{*, n}=0 .
$$

By Theorem 1.1, $\exists C>0$ such that whenever $0<\lambda<C /\|F\|_{*, n}$ we have

$$
\sup _{l(Q) \leq 2^{-n}} \frac{1}{|Q|} \int_{Q} e^{\lambda\left|F-F_{Q}\right|}<2 .
$$

Hence $\bar{\mu}_{n}^{*}(F) \leq\|F\|_{*, n} / C$. Since $\bar{\mu}_{n}(F) \leq \bar{\mu}_{n}^{*}(F)$ and $\mu_{n}(f) \leq \bar{\mu}_{n}(F)$, it follows that $\mu_{n}(f) \leq C$ for $n=0,1,2, \ldots$ and $\lim _{n \rightarrow \infty} \mu_{n}(f)=0$ and this proves $(1) \Rightarrow(2)$.

Proof of $(2) \Rightarrow(1)$ :

Part (i): Extension to dyadic-VMO.

Condition (2) in Theorem I implies there exists a sequence $\left\{\lambda_{n}\right\}_{n \geq 0}$, $0<\lambda_{n} \uparrow \infty$ such that

$$
\sup _{l(Q) \leq 2^{-n}} \frac{1}{|Q|} \int_{Q \cap E} e^{\lambda_{n}\left|f-f_{Q \cap E}\right|}<2 .
$$

Let $n_{0}=0$ and define a sequence $\left\{n_{k}\right\}_{k \geq 1} \subseteq \mathbf{N}$ by the condition $\lambda_{n}>2 \lambda_{n_{k}}$ if and only if $n \geq n_{k+1}$. To simplify the notation we will write $\lambda_{k}$ for $\lambda_{n_{k}}$. Now define for each $k \geq 0$

$$
\mathfrak{F}_{k}=\left\{Q: Q \text { dyadic, } Q \subseteq Q_{0}, l(Q) \geq 2^{-n_{k}}\right\} \text {. }
$$

For each $n=0,1,2, \ldots$ we define $f_{n}=\sum_{Q \in D_{n}} f_{Q \cap E} \chi_{Q \cap E}$.

The idea of the proof is as follows: We write

$$
f=f_{0}+\sum_{k \geq 0}\left(f_{n_{k+1}}-f_{n_{k}}\right)
$$

and note that in order to extend $f$ it suffices to extend each of the functions $f_{0}, f_{n_{k+1}}-f_{n_{k}}, k \geq 0$. These functions are constant at every point of $E$ which belong to the same small dyadic cube and so 
we would like to extend each of these functions to dyadic-BMO functions which are constant on small dyadic cubes and in particular will therefore be in dyadic-VMO. To do this we proceed as in the proof of Theorem 2.2; that is, we first show (in Lemma 3.1) that

$$
e^{\left(\lambda_{0} / 4\right) f_{0}} \in A_{2}\left(E, \mathfrak{F}_{0}\right) \quad \text { and } \quad e^{\lambda_{k / 4}\left(f_{n_{k+1}}-f_{n_{k}}\right)} \in A_{2}\left(E, \mathfrak{F}_{k+1}\right)
$$

and satisfy a reverse Hölder inequality. We then factor and extend these weights using a truncated dyadic maximal function instead of the full dyadic maximal function. We do this to ensure that the $A_{1}\left(Q_{0}, \mathfrak{F}_{k}\right)$-weights we obtain using Lemma 2.1 (that is, the weights $W_{1}, W_{2}$ in the notation of Theorem 2.2) will be constant on small dyadic cubes. The difficulty that now arises is that we cannot conclude as in Theorem 2.2 that the quotient of each factored $A_{1}\left(E, \mathfrak{F}_{k}\right)$ weight with the corresponding truncated maximal function is uniformly bounded on $E$. To accommodate this possibly large quotient into an appropriate VMO function we will appeal to Lemma 2.2. We proceed with the dyadic extension:

LEMMA 3.1. There exists $C>0$, depending only on the dimension, such that for all $k \geq 0$,

$$
\sup _{Q \in \mathfrak{F}_{k+1}}\left(\frac{1}{|Q|} \int_{Q \cap E} e^{\left(\lambda_{k} / 2\right)\left(f_{n_{k+1}}-f_{n_{k}}\right)}\right)\left(\frac{1}{|Q|} \int_{Q \cap E} e^{-\left(\lambda_{k} / 2\right)\left(f_{n_{k+1}}-f_{n_{k}}\right)}\right) \leq C,
$$

$$
\sup _{Q \in \mathfrak{F}_{0}}\left(\frac{1}{|Q|} \int_{Q \cap E} e^{\left(\lambda_{0} / 2\right) f_{0}}\right)\left(\frac{1}{|Q|} \int_{Q \cap E} e^{-\left(\lambda_{0} / 2\right) f_{0}}\right) \leq C
$$

Proof. Let $Q$ be a dyadic cube with $l(Q) \geq 2^{-n_{J}}$ and let $\left\{Q_{i}\right\}$ be those dyadic subcubes of $Q$ of length $2^{-n_{j}}$. Then for all $\lambda \leq \lambda_{j}$,

$$
\begin{aligned}
\frac{1}{|Q|} \int_{Q \cap E} e^{\lambda\left|f-f_{n_{j}}\right|} & =\sum \frac{\left|Q_{i}\right|}{|Q|}\left(\frac{1}{\left|Q_{i}\right|} \int_{Q_{i} \cap E} e^{\lambda\left|f-f_{n_{j}}\right|}\right) \\
& \leq \sum \frac{\left|Q_{i}\right|}{|Q|}\left(\frac{1}{\left|Q_{i}\right|} \int_{Q_{\imath} \cap E} e^{\lambda\left|f-f_{Q_{i} \cap E}\right|}\right) \leq C
\end{aligned}
$$

and hence

$$
\frac{1}{|Q|} \int_{Q \cap E} e^{\lambda\left(f-f_{n_{J}}\right)} \frac{1}{|Q|} \int_{Q \cap E} e^{-\lambda\left(f-f_{n_{J}}\right)} \leq C
$$


Now if $Q$ is a dyadic cube with $l(Q) \leq 2^{-n_{\jmath}}$ then for all $\lambda \leq \lambda_{j}$ we are given

$$
\frac{1}{|Q|} \int_{Q \cap E} e^{\lambda\left(f-f_{Q \cap E}\right)} \frac{1}{|Q|} \int_{Q \cap E} e^{-\lambda\left(f-f_{Q \cap E}\right)} \leq C
$$

and hence

$$
\left(\frac{1}{|Q|} \int_{Q \cap E} e^{\lambda f}\right)\left(\frac{1}{|Q|} \int_{Q \cap E} e^{-\lambda f}\right) \leq C .
$$

We note that if $j=0,(3.2)$ holds for all $Q \in \mathfrak{F}_{0}$ and for all $\lambda \leq \lambda_{0}$. Now (3.2) implies that for all $Q \in \mathfrak{F}_{j+1}, l(Q) \leq 2^{-n_{\jmath}}$ and for all $\lambda \leq \lambda_{j}$

$$
\frac{1}{|Q|} \int_{Q \cap E} e^{\lambda\left(f-f_{n_{J}}\right)} \frac{1}{|Q|} \int_{Q \cap E} e^{-\lambda\left(f-f_{n_{J}}\right)} \leq C .
$$

Since

$$
\begin{aligned}
& \frac{1}{|Q|} \int_{Q \cap E} e^{(\lambda / 2)\left(f_{n_{k+1}}-f_{n_{k}}\right)} \\
& \quad \leq\left(\frac{1}{|Q|} \int_{Q \cap E} e^{\lambda\left(f-f_{n_{k}}\right)}\right)^{1 / 2}\left(\frac{1}{|Q|} \int_{Q \cap E} e^{-\lambda\left(f-f_{n_{k+1}}\right)}\right)^{1 / 2}
\end{aligned}
$$

(and similarly for $\left.\frac{1}{|Q|} \int_{Q \cap E} e^{-(\lambda / 2)\left(f_{n_{k+1}}-f_{n_{k}}\right)}\right)$ we see that (1) follows from (3.1) and (3.3).

Now (3.1), (3.2) and Hölder's inequality imply that for all $\lambda \leq \lambda_{0} / 2$ and for all $Q \in \mathfrak{F}_{0}$

$$
\left(\frac{1}{|Q|} \int_{Q \cap E} e^{\lambda f_{0}}\right)\left(\frac{1}{|Q|} \int_{Q \cap E} e^{-\lambda f_{0}}\right) \leq C
$$

and this gives (2) which completes the proof of the lemma.

To simplify the notation we set $M_{k}(g)=M_{\mathfrak{F}_{k}}(g)$ and $m_{k}(g)=$ $m_{\mathfrak{F}_{h}}(g)$. Corollary 2.1 implies the following:

For each $k=0,1,2, \ldots$ there exist $u_{k}, v_{k} \in A_{1}\left(E, \mathfrak{F}_{k}\right)$ such that

$$
\frac{u_{0}}{v_{0}}=\exp \left(\frac{\lambda_{0}}{4} f_{0}\right)
$$

and

$$
\frac{u_{k}}{v_{k}}=\exp \left(\frac{\lambda_{k-1}}{4}\left(f_{n_{k}}-f_{n_{k-1}}\right)\right) \quad \forall k=1,2, \ldots
$$

Furthermore, since the $A_{2}\left(E, \mathfrak{F}_{k}\right)$-weights and the maximal function $m_{k}(\cdot)$ are constant at every point of $E$ which belong to the same dyadic cube of length $2^{-n_{k}}$, the proof of Corollary 2.1 given in [2] shows that the same is true for the $A_{1}\left(E, \mathfrak{F}_{k}\right)$-weights $u_{k}$ and $v_{k}$. 
Now for each $k=0,1,2, \ldots$ and for each $x \in \mathbf{R}^{d}$ we define

$$
\begin{gathered}
U_{k}(x)=M_{k}\left(\chi_{E} u_{k}\right)(x), \\
V_{k}(x)=M_{k}\left(\chi_{E} v_{k}\right)(x) .
\end{gathered}
$$

Then, for all $x \in E$,

$$
\exp \left(\frac{\lambda_{k-1}}{4}\left(f_{n_{k}}-f_{n_{k-1}}\right)(x)\right)=\frac{U_{k}(x)}{V_{k}(x)} w_{k}(x) \quad \forall k \geq 1
$$

and

$$
\exp \left(\frac{\lambda_{0}}{4} f k_{0}(x)\right)=\frac{U_{0}(x)}{V_{0}(x)} w_{0}(x)
$$

where

$$
w_{k}=\frac{u_{k}}{v_{k}} \frac{m_{k}\left(v_{k}\right)}{m_{k}\left(u_{k}\right)} \quad \forall k \geq 0
$$

Hence,

$$
f_{n_{k}}-f_{n_{k-1}}=\frac{8}{\lambda_{k-1}} \log \left(\frac{U_{k}}{V_{k}}\right)^{1 / 2}+\frac{4}{\lambda_{k-1}} \log w_{k}
$$

and

$$
f_{0}=\frac{8}{\lambda_{0}} \log \left(\frac{U_{0}}{V_{0}}\right)^{1 / 2}+\frac{4}{\lambda_{0}} \log w_{0} .
$$

Now Lemma 2.1 implies $\exists C>0$ such that

$$
M_{k}\left(U_{k}^{1 / 2}\right) \leq C U_{k}^{1 / 2} \text { and } M_{k}\left(V_{k}^{1 / 2}\right) \leq C V_{k}^{1 / 2}
$$

and so by Proposition 2.1 (ii), $\left(U_{k} / V_{k}\right)^{1 / 2} \in A_{2}\left(Q_{0}, \mathfrak{F}_{k}\right)$.

Then, by Proposition 2.1 (iii), we conclude that

$$
\sup _{Q \in \mathfrak{F}_{k}} \frac{1}{|Q|} \int_{Q}\left|\log \left(\frac{U_{k}}{V_{k}}\right)-\left(\log \left(\frac{U_{k}}{V_{k}}\right)\right)_{Q}\right| \leq C .
$$

In particular since $U_{k}, V_{k}$ are constant on dyadic cubes of length $2^{-n_{k}}$, we have $\log \left(U_{k} / V_{k}\right) \in$ dyadic- $\operatorname{VMO}\left(Q_{0}\right)$.

Finally we need to extend the functions $\log w_{k}$ to dyadic-VMO $\left(Q_{0}\right)$. Let $Q_{k}$ be a dyadic cube of length $2^{-n_{k}}$ with $\left|Q_{k} \cap E\right|>0$. Then since $u_{k}$ and $v_{k}$ are constant on $Q_{k} \cap E$ we have for all $x \in Q_{k} \cap E$

$$
u_{k}(x) \leq \frac{1}{\left|Q_{k} \cap E\right|} \int_{Q_{k} \cap E} u_{k}(t) d t \leq \frac{\left|Q_{k}\right|}{\left|Q_{k} \cap E\right|} m_{k}\left(u_{k}\right)(x)
$$

and similarly

$$
v_{k}(x) \leq \frac{\left|Q_{k}\right|}{\left|Q_{k} \cap E\right|} m_{k}\left(v_{k}\right)(x)
$$


and hence

$$
\left|\log w_{k}(x)\right| \leq \log C+\log \frac{\left|Q_{k}\right|}{\left|Q_{k} \cap E\right|} .
$$

Hence by Lemma 2.2, there exists $h_{k} \in$ dyadic- $\operatorname{VMO}\left(Q_{0}\right)$ with $\operatorname{supp}\left(h_{k}\right) \subseteq Q_{k}$ satisfying

$$
\begin{gathered}
\left|\log w_{k}(x)-h_{k}(x)\right| \leq C, \quad \forall x \in Q_{k} \cap E \quad \text { and } \\
\sup _{l(Q) \geq l\left(Q_{k}\right)} \frac{1}{|Q|} \int_{Q} h_{k}(t) d t \leq C .
\end{gathered}
$$

We now define

$$
H_{k}(x)= \begin{cases}h_{k}(x) & \text { if } x \in Q_{k}, Q_{k} \in D_{n_{k}},\left|Q_{k} \cap E\right|>0, \\ 0 & \text { otherwise. }\end{cases}
$$

It is easy to check $H_{k} \in$ dyadic- $\operatorname{VMO}\left(Q_{0}\right)$ with $\left\|H_{k}\right\|_{*, d} \leq C$ and $\left|\log w_{k}(x)-H_{k}(x)\right| \leq C, \forall x \in E$. This implies $\exists R_{k}(x) \in L_{\infty}\left(\mathbf{R}^{d}\right)$ with $\left\|R_{k}\right\|_{\infty} \leq C$ and which is constant on dyadic cubes of length $2^{-n_{k}}$ and satisfies $R_{k}(x)=\log w_{k}(x)-H_{k}(x), \forall x \in E$. In particular, $R_{k} \in$ dyadic- $\operatorname{VMO}\left(Q_{0}\right)$ with $\left\|R_{k}\right\|_{*, d} \leq C, \forall k$. Hence the function $W_{k}=\exp \left(R_{k}+H_{k}\right)$ satisfies $\log W_{k} \in$ dyadic- $\operatorname{VMO}\left(Q_{0}\right),\left\|\log W_{k}\right\|_{*, d} \leq$ $C$ and $W_{k} \chi_{E}=w_{k}$.

Now define

$$
F(x)= \begin{cases}\sum_{k \geq 0} \frac{2}{\lambda_{k-1}}\left(G_{k}+R_{k}+H_{k}\right) & \text { if } x \in Q_{0} \\ 0 & \text { otherwise }\end{cases}
$$

where $G_{k}=4 \log \left(U_{k} / V_{k}\right)^{1 / 2}$ and by $\lambda_{-1}$ we mean $\lambda_{0}$. Since $G_{k}+$ $R_{k}+H_{k} \in$ dyadic- $\operatorname{VMO}\left(Q_{0}\right)$ with $\left\|G_{k}+R_{k}+H_{k}\right\|_{*, d} \leq C$ and since $\sum_{k \geq 0} 1 /\left(\lambda_{k-1}\right)<\infty$, it follows that $F \in$ dyadic-VMO and $\|F\|_{*, d} \leq C$.

\section{Furthermore}

$$
\sum_{k=0}^{\infty} \frac{2}{\lambda_{k-1}}\left(G_{k}+R_{k}+H_{k}\right) \chi_{E}=\sum_{k \geq 0}\left(f_{n_{k+1}}-f_{n_{k}}\right)+f_{0}=f \quad \text { a.e. on } E \text {. }
$$

Hence $F$ is a dyadic-VMO extension of $f$.

Part (ii): Extension to non-dyadic VMO.

If $S$ is a set in $\mathbf{R}^{d}$ we will denote by $S-\alpha$ the set $\{x-\alpha: x \in S\}$.

For each $\alpha \in \frac{1}{3} Q_{0}$ the function $f^{(\alpha)}(x)=f(x-\alpha)$ satisfies the hypothesis of Theorem I with the set $E$ replaced by $E-\alpha$. Hence by the proof in part (i) above, there exists $F^{(\alpha)} \in$ dyadic-VMO with 
$\left\|F^{(\alpha)}\right\|_{*} \leq C, F^{(\alpha)}=f^{(\alpha)}$ a.e. on $E-\alpha$ and $\operatorname{supp}\left(F^{(\alpha)}\right) \subseteq Q_{0}$. We claim that the function

$$
F(x)=3^{d} \int_{\alpha \in Q_{0} / 3} F^{(\alpha)}(x+\alpha) d \alpha
$$

is a VMO extension of $f$. It is clear that $F=f$ a.e. on $E$. It will follow from Lemma 3.2 below that $F \in \mathrm{VMO}$.

Lemma 3.2. Let $Q$ be a cube and let $\left\{f^{(\alpha)}: \alpha \in Q\right\}$ be a collection of dyadic-VMO functions satisfying

(1) $\sup _{\alpha \in Q}\left\|f^{(\alpha)}\right\|_{*, d} \leq 1$,

(2) there exists $r>0$ such that for all $\alpha \in Q, \operatorname{supp}\left(f^{(\alpha)}\right) \subseteq 2^{r} Q$.

Then the function

$$
f(x)=\frac{1}{|Q|} \int_{\alpha \in Q} f^{(\alpha)}(x+\alpha) d \alpha
$$

belongs to VMO with $\|f\|_{*} \leq C_{r}$ where $C_{r}$ depends only on $r$ and the dimension.

REMARKs. (1) The idea of averaging dyadic-BMO comes from [6] where the BMO version of Lemma 3.2 is stated (and proved implicitly). Our argument is different to that used in [6] and the simpler BMO version of our argument can be used to provide an easy proof of the theorem in [6].

(2) Let $\operatorname{BMO}(\rho)$ denote the space of those VMO functions whose mean oscillation over any cube is $O(\rho(l(Q)))$ where $\rho$ is a positive, non-decreasing function with $\rho(0+)=0$. In the case when each $f^{(\alpha)}$ belongs to $\operatorname{BMO}(\rho)$, Lemma 3.2 may be deduced from the results in $\S 3$ of [13]; the conclusion in this case being that $f \in \operatorname{BMO}(\tilde{\rho})$ where $\tilde{\rho}(t)=t \int_{t}^{\infty} \rho(\theta) / \theta^{2} d \theta$. The argument in [13] is a version of the averaging argument in [6]. The proof below can be easily modified to obtain this result.

Proof of Lemma 3.2. Without loss of generality we may assume $Q=$ $Q_{0}$, the unit cube in $\mathbf{R}^{d}$.

We first show that for each $\varepsilon>0$ there exists $\delta>0$ such that if $Q$ is any cube with $l(Q)<\delta$ then

$$
\frac{1}{|Q|} \int_{Q}\left|f-f_{Q}\right|<\varepsilon
$$


Fix $0<\varepsilon<\frac{1}{2}$. For each $\alpha \in Q_{0}, f^{(\alpha)} \in$ dyadic-VMO and hence there exists $n_{\alpha} \in \mathbf{N}$ so that

$$
\frac{1}{|Q|} \int_{Q}\left|f^{(\alpha)}-\left(f^{(\alpha)}\right)_{Q}\right|<\varepsilon^{d+1}
$$

whenever $Q$ is a dyadic cube of length $\leq 2^{-n_{\alpha}}$. Hence by Egoroff's theorem there exists $N_{1} \in \mathbf{N}$ so that the set

$$
\begin{aligned}
S_{0}=\left\{\alpha \in Q_{0}:\right. & \frac{1}{|Q|} \int_{Q}\left|f^{(\alpha)}-\left(f^{(\alpha)}\right)_{Q}\right|<\varepsilon^{d+1} \\
& \text { whenever } \left.Q \text { is a dyadic cube of length } \leq 2^{-N_{1}}\right\}
\end{aligned}
$$

has measure $\geq 1-\varepsilon$. Choose $N_{2}$ so that $N_{2} 2^{-N_{2}}<\varepsilon 2^{-N_{1}}$ and let $Q$ be any cube with $l(Q) \leq 2^{-N_{2}}$. Let

$S_{1}=\left\{\alpha \in Q_{0}: Q-\alpha\right.$ is contained in a dyadic cube of length $\left.\leq \frac{1}{\varepsilon} l(Q)\right\}$ and note that $\left|Q_{0} \cap S_{1}^{c}\right| \leq C_{\varepsilon}$.

If $\alpha \in S_{0} \cap S_{1}$ we claim that

$$
\frac{1}{|Q|} \int_{Q}\left|f^{(\alpha)}(x+\alpha)-f^{(\alpha)}(\cdot+\alpha)_{Q}\right| d x \leq 2 \varepsilon .
$$

To see this let $Q_{1}$ be that dyadic cube of length $\leq \frac{1}{\varepsilon} l(Q)$ that contains $Q-\alpha$. Then,

$$
\begin{aligned}
& \frac{1}{|Q|} \int_{Q}\left|f^{(\alpha)}(x+\alpha)-\left(f^{(\alpha)}(\cdot+\alpha)\right)_{Q}\right| d x \\
& \quad \leq 2 \inf _{a \in \mathbf{R}} \frac{1}{|Q|} \int_{Q}\left|f^{(\alpha)}(x+\alpha)-a\right| d x \\
& \quad \leq 2 \frac{1}{|Q|} \int_{Q}\left|f^{(\alpha)}(x+\alpha)-\left(f^{(\alpha)}\right)_{Q_{1}}\right| d x \\
& \quad \leq 2 \frac{\left|Q_{1}\right|}{|Q|} \frac{1}{|Q|} \int_{Q_{1}}\left|f^{(\alpha)}(x)-\left(f^{(\alpha)}\right)_{Q_{1}}\right| d x \leq 2 \varepsilon^{-d} \varepsilon^{d+1}=2 \varepsilon .
\end{aligned}
$$

For each $k \geq 0$ let $f_{k}^{(\alpha)}=\sum_{Q \in D_{k}}\left(f^{(\alpha)}\right)_{Q} \chi_{Q}$ and choose $m$ so that $2^{-(m+1)} \leq l(Q)<2^{-m}$. Let

$$
g_{1}^{(\alpha)}=f^{(\alpha)}-f_{m}^{(\alpha)} \text { and } g_{2}^{(\alpha)}=f_{m}^{(\alpha)}
$$

Now for all $\alpha \in Q_{0}$,

$$
\begin{aligned}
& \frac{1}{|Q|} \int_{Q}\left|g_{1}^{(\alpha)}(x+\alpha)\right| d x \leq C 2^{m d} \int_{Q-\alpha}\left|f^{(\alpha)}(x)-f_{m}^{(\alpha)}(x)\right| d x \\
& \quad \leq C \sum \frac{1}{\left|Q_{i}\right|} \int_{Q_{l}}\left|f^{(\alpha)}-\left(f^{(\alpha)}\right)_{Q_{l}}\right| d x
\end{aligned}
$$


where the sum is taken over those dyadic cubes $Q_{i}$ of length $2^{-m}$ which intersect $Q-\alpha$. If $\alpha \in S_{0}$ then for each such $Q_{i}$,

$$
\frac{1}{\left|Q_{i}\right|} \int_{Q_{i}}\left|f^{(\alpha)}-\left(f^{(\alpha)}\right)_{Q_{\imath}}\right|<\varepsilon^{d+1}
$$

while if $\alpha \in Q_{0} \cap S_{0}^{c}$

$$
\frac{1}{\left|Q_{i}\right|} \int_{Q_{i}}\left|f^{(\alpha)}-\left(f^{(\alpha)}\right)_{Q_{\imath}}\right| \leq 1
$$

Since there are at most a fixed number of such cubes we have

$$
\frac{1}{|Q|} \int_{Q}\left|g_{1}^{(\alpha)}(x+\alpha)\right| d x \leq \begin{cases}C \varepsilon & \text { if } \alpha \in S_{0} \\ C & \text { if } \alpha \in Q_{0} \cap S_{0}^{c} .\end{cases}
$$

Now for each $0 \leq j \leq m$ let

$$
A_{j}=\left\{\alpha \in Q_{0}: Q-\alpha \text { is contained in a dyadic cube of length } 2^{-j}\right\}
$$

and note that $\left|A_{j}^{c}\right| \leq C 2^{j-m}$.

Since $\operatorname{supp}\left(f^{(\alpha)}\right) \subseteq 2^{r} Q_{0}$, and $\left\|f^{(\alpha)}\right\|_{*} \leq 1$, it follows that there exists $C>0$ depending only on $r$ and the dimension such that if $\alpha \in\left(A_{j} \cap A_{j+1}^{c}\right) \cap S_{0}$ then

$$
\sup _{x, y \in Q-\alpha}\left|g_{2}^{(\alpha)}(x+\alpha)-g_{2}^{(\alpha)}(y+\alpha)\right| \leq \begin{cases}C \varepsilon(m-j) & \text { if } j \geq N_{1}, \\ C(m-j) & \text { if } 0 \leq j<N_{1},\end{cases}
$$

and if $\alpha \in A_{0}^{c} \cap S_{0}$ then

$$
\sup _{x, y \in Q-\alpha}\left|g_{2}^{(\alpha)}(x+\alpha)-g_{2}^{(\alpha)}(y+\alpha)\right| \leq C(m+r)
$$

while if $\alpha \in\left(A_{j} \cap A_{j+1}^{c}\right) \cap S_{0}^{c}$

$$
\sup _{x, y \in Q-\alpha}\left|g_{2}^{(\alpha)}(x+\alpha)-g_{2}^{(\alpha)}(y+\alpha)\right| \leq C(m-j)
$$

and if $\alpha \in A_{0}^{c} \cap S_{0}^{c}$ then

$$
\sup _{x, y \in Q-\alpha}\left|g_{2}^{(\alpha)}(x+\alpha)-g_{2}^{(\alpha)}(y+\alpha)\right| \leq C(m+r) .
$$

Hence if $j \geq N_{1}$ then

$$
\begin{aligned}
& \int_{A, \cap A_{j+1}^{c} \cap S_{0}} \sup _{x, y \in Q-\alpha}\left|g_{2}^{(\alpha)}(x+\alpha)-g_{2}^{(\alpha)}(y+\alpha)\right| d \alpha \\
& \leq C \varepsilon(m-j) 2^{j-m}
\end{aligned}
$$


and if $0 \leq j<N_{1}$ then

$$
\begin{aligned}
& \int_{A_{j} \cap A_{j+1}^{c} \cap S_{0}} \sup _{x, y \in Q-\alpha}\left|g_{2}^{(\alpha)}(x+\alpha)-g_{2}^{(\alpha)}(y+\alpha)\right| d \alpha \\
& \leq C(m-j) 2^{j-m}
\end{aligned}
$$

Also

$$
\int_{A_{0}^{c} \cap S_{0}} \sup _{x, y \in Q-\alpha}\left|g_{2}^{(\alpha)}(x+\alpha)-g_{2}^{(\alpha)}(y+\alpha)\right| d \alpha \leq C(m+r) 2^{-m}
$$

and

$$
\begin{aligned}
& \int_{A_{j} \cap A_{j+1}^{c} \cap S_{0}^{c}} \sup _{x, y \in Q-\alpha}\left|g_{2}^{(\alpha)}(x+\alpha)-g_{2}^{(\alpha)}(y+\alpha)\right| d \alpha \\
& \leq C(m-j) \min \left(2^{j-m}, \varepsilon\right), \\
& \int_{A_{0}^{c} \cap S_{0}^{c}} \sup _{x, y \in Q-\alpha}\left|g_{2}^{(\alpha)}(x+\alpha)-g_{2}^{(\alpha)}(y+\alpha)\right| d \alpha \\
& \leq C(m+r) \min \left(2^{-m}, \varepsilon\right) .
\end{aligned}
$$

\section{Hence}

(3.7) $\int_{Q_{0} \backslash\left(S_{0} \cap S_{1}\right)} \sup _{x, y \in Q}\left|g_{2}^{(\alpha)}(x+\alpha)-g_{2}^{(\alpha)}(y+\alpha)\right| d \alpha$

$$
\begin{aligned}
\leq & \sum_{j=0}^{m} C(m-j) \min \left(2^{j-m}, \varepsilon\right)+C(m+r) \min \left(2^{-m}, \varepsilon\right) \\
& +\sum_{j=N_{1}}^{m} C \varepsilon(m-j) 2^{j-m} \\
& +\sum_{j=0}^{N_{1}} C(m-j) 2^{j-m}+C(m+r) 2^{-m} \\
\leq & C \varepsilon \log \frac{1}{\varepsilon}+C m 2^{-m}+C \varepsilon+\left(m-N_{1}\right) 2^{N_{1}-m}+C m 2^{-m} \\
\leq & C \varepsilon \log \frac{1}{\varepsilon}+C \varepsilon+C\left(m 2^{-m}\right) 2^{N_{1}} \\
\leq & C \varepsilon \log \frac{1}{\varepsilon}+C \varepsilon+C \varepsilon \leq C \varepsilon \log \frac{1}{\varepsilon} .
\end{aligned}
$$


Combining (3.5), (3.6) and (3.7) we get

$$
\begin{aligned}
& \frac{1}{|Q|} \int_{Q}\left|f-f_{Q}\right| d x \\
& \leq \int_{Q_{0}}\left(\frac{1}{|Q|} \int_{Q}\left|f^{(\alpha)}(x+\alpha)-\left(f^{(\alpha)}(\cdot+\alpha)\right)_{Q}\right| d x\right) d \alpha \\
& \leq \int_{S_{0} \cap S_{1}}\left(\frac{1}{|Q|} \int_{Q}\left|f^{(\alpha)}(x+\alpha)-\left(f^{(\alpha)}(\cdot+\alpha)\right)_{Q}\right| d x\right) d \alpha \\
&+\int_{Q_{0} \backslash\left(S_{0} \cap S_{1}\right)} \sup _{x, y \in Q}\left|g_{2}^{(\alpha)}(x+\alpha)-g_{2}^{(\alpha)}(y+\alpha)\right| d \alpha \\
&+2 \int_{S_{0}}\left(\frac{1}{|Q|} \int_{Q}\left|g_{1}^{(\alpha)}(x+\alpha)\right| d x\right) d \alpha \\
&+2 \int_{Q_{0} \backslash S_{0}}\left(\frac{1}{|Q|} \int_{Q}\left|g_{1}^{(\alpha)}(x+\alpha)\right| d x\right) d \alpha \\
& \leq C \varepsilon+C \varepsilon \log \frac{1}{\varepsilon}+C \varepsilon+C \varepsilon \leq C \varepsilon \log \frac{1}{\varepsilon} .
\end{aligned}
$$

It remains to show that

$$
\sup _{Q} \frac{1}{|Q|} \int_{Q}\left|f-f_{Q}\right|<\infty .
$$

It is clear from what we established above that it suffices to prove (3.8) when the supremum is taken over all cubes with $l(Q) \geq 1$. Fix $Q$ with $l(Q) \geq 1$. Then for all $\alpha \in Q_{0}$,

$$
\begin{aligned}
& \frac{1}{|Q|} \int_{Q}\left|f^{(\alpha)}(x+\alpha)\right| d x \\
& \quad \leq \frac{1}{|Q|} \int_{Q}\left|f_{0}^{(\alpha)}(x+\alpha)\right| d x+\frac{1}{|Q|} \int_{Q}\left|f^{(\alpha)}-f_{0}^{(\alpha)}\right| d x .
\end{aligned}
$$

Since each $f^{(\alpha)}$ is supported on $2^{r} Q_{0}$ there exists $C>0$, depending only on $r$ and the dimension, such that $\left|f_{0}^{(\alpha)}(x)\right| \leq C$ for all $x \in \mathbf{R}^{d}$. Hence

$$
\frac{1}{|Q|} \int_{Q}\left|f_{0}^{(\alpha)}(x+\alpha)\right| d x \leq C .
$$

Let $\left\{Q_{i}\right\}$ be those dyadic cubes of length 1 with $Q_{i} \cap(Q-\alpha) \neq \varnothing$. Then for all $\alpha \in Q_{0}$,

$$
\begin{aligned}
& \frac{1}{|Q|} \int_{Q}\left|f^{(\alpha)}(x+\alpha)-f_{0}^{(\alpha)}(x+\alpha)\right| d x \\
& \quad \leq C \sum_{Q_{l}} \frac{1}{\left|Q_{i}\right|} \int_{Q_{l}}\left|f^{(\alpha)}(x)-\left(f^{(\alpha)}\right)_{Q_{\imath}}\right| d x \leq C .
\end{aligned}
$$


Hence

$$
\begin{aligned}
& \frac{1}{|Q|} \int_{Q}\left|f-f_{Q}\right| \leq C \frac{1}{|Q|} \int_{Q}|f| \\
& \quad \leq C \int_{Q_{0}}\left(\frac{1}{|Q|} \int_{Q}\left|f^{(\alpha)}(x+\alpha)\right| d x\right) d \alpha \leq C .
\end{aligned}
$$

and (3.8) now follows. This completes the proof of Lemma 3.2 and Theorem I.

A consequence of Theorem I which has useful applications is the following corollary:

COROLlary 3.1. Let $E_{1}, E_{2}$ be measurable subsets of the unit cube in $\mathbf{R}^{d}$ and suppose there exists an increasing sequence of positive numbers $\left\{\lambda_{n}\right\}_{n=0}^{\infty}$ with $\lambda_{n} \rightarrow \infty$ such that for each $n \in \mathbf{N}$ and for each cube $Q$ with $l(Q) \leq 2^{-n}$ we have

$$
\min \left(\frac{\left|Q \cap E_{1}\right|}{|Q|}, \frac{\left|Q \cap E_{2}\right|}{|Q|}\right)<e^{-\lambda_{n}}
$$

Then there exists $F \in \mathrm{VMO},\|F\|_{*} \leq C_{\lambda_{0}}$ with $F=0$ on $E_{1}$ and $F=1$ on $E_{2}$.

Proof. Set $E=E_{1} \cup E_{2}$ in Theorem I and define

$$
f(x)= \begin{cases}0 & \text { if } x \in E_{1}, \\ 1 & \text { if } x \in E_{2},\end{cases}
$$

and

$$
a_{Q}= \begin{cases}1 & \text { if }\left|Q \cap E_{1}\right| /|Q|<e^{-\lambda_{n}} \\ 0 & \text { otherwise }\end{cases}
$$

Acknowledgment. The material in this paper formed part of my thesis dissertation at the California Institute of Technology. I am very grateful to my thesis advisor, Professor Tom Wolff for his help and encouragement.

\section{REFERENCES}

[1] M. Christ and R. Fefferman, A note on weighted norm inequalities for the HardyLittlewood maximal operator, Proc. Amer. Math. Soc., 87 (1983), 447-448.

[2] R. Coifman, P. Jones and J. Rubio de Francia, On a constructive decomposition of BMO functions and factorization of $A_{p}$ weights, Proc. Amer. Math. Soc., 87 (1983), 675-676. 
[3] R. Coifman and R. Rochberg, Another characterization of BMO, Proc. Amer. Math. Soc., 79 (1980), 249-254.

[4] J. Garnett, Bounded Analytic Functions, Academic Press, New York, 1980.

[5] J. Garnett and P. Jones, The distance in BMO to $L_{\infty}$, Ann. of Math., 108 (1978), 373-393.

[6] _- BMO from dyadic BMO, Pacific J. Math., 99 (1982), 351-372.

[7] F. John and L. Nirenberg, On functions of bounded mean oscillation, Comm. Pure and Applied Math., 14 (1961), 415-426.

[8] P. Jones, Factorization of $A_{p}$ weights, Ann. of Math., 111 (1980), 511-530.

[9] B. Muchenhoupt, Weighted norm inequalities for the Hardy maximal function, Trans. Amer. Math. Soc., 165 (1972), 207-226.

[10] J. Rubio de Francia, Factorization and extrapolation of weights, Bull. Amer. Math. Soc., 7 (1982), 393-395.

[11] D. Sarason, Functions of vanishing mean oscillation, Trans. Amer. Math. Soc., 207 (1975), 391-405.

[12] __ Function theory on the unit circle, Virginia Poly. Inst. and State Univ., Blacksburg, Virginia.

[13] W. Smith, $\operatorname{BMO}(\rho)$ and Carleson measures, Trans. Amer. Math. Soc., 287 (1985), 107-126.

[14] T. Wolff, Two algebras of bounded functions, Duke Math. J., 49 (1982), 321328.

[15] _ Restrictions of $A_{p}$ weights, preprint.

Received April 12, 1988 and in revised form August 22, 1988.

FLORIDA INTERNATIONAL UNIVERSITY

MiAMI, FL 33199 



\section{PACIFIC JOURNAL OF MATHEMATICS EDITORS}

\author{
V. S. VARADARAJAN \\ (Managing Editor) \\ University of California \\ Los Angeles, CA 90024-1555-05 \\ Herbert Clemens \\ University of Utah \\ Salt Lake City, UT 84112 \\ ThOMAs EnRIGHT \\ University of California, San Diego \\ La Jolla, CA 92093
}

R. FINN

Stanford University

Stanford, CA 94305

HeRmanN FlaschKa

University of Arizona

Tucson, AZ 85721

VAUGHAN F. R. Jones

University of California

Berkeley, CA 94720

SteVEn KerckHofF

Stanford University

Stanford, CA 94305

\author{
ROBION KIRBY \\ University of California \\ Berkeley, CA 94720 \\ C. C. MOORE \\ University of California \\ Berkeley, CA 94720 \\ HAROLd StaRK \\ University of California, San Diego \\ La Jolla, CA 92093
}

\section{ASSOCIATE EDITORS}
R. ARENS
E. F. BECKENBACH
B. H. NeUMANN
F. WoLF
K. YoshidA (1906-1982)
(1904-1989)

\section{SUPPORTING INSTITUTIONS}

\section{UNIVERSITY OF ARIZONA UNIVERSITY OF BRITISH COLUMBIA CALIFORNIA INSTITUTE OF TECHNOLOGY UNIVERSITY OF CALIFORNIA MONTANA STATE UNIVERSITY UNIVERSITY OF NEVADA, RENO NEW MEXICO STATE UNIVERSITY OREGON STATE UNIVERSITY}

\author{
UNIVERSITY OF OREGON \\ UNIVERSITY OF SOUTHERN CALIFORNIA \\ STANFORD UNIVERSITY \\ UNIVERSITY OF HAWAII \\ UNIVERSITY OF TOKYO \\ UNIVERSITY OF UTAH \\ WASHINGTON STATE UNIVERSITY \\ UNIVERSITY OF WASHINGTON
}

The Supporting Institutions listed above contribute to the cost of publication of this Journal, but they are not owners or publishers and have no responsibility for its content or policies.

Mathematical papers intended for publication in the Pacific Journal of Mathematics should be in typed form or offset-reproduced (not dittoed), double spaced with large margins. Please do not use built up fractions in the text of the manuscript. However, you may use them in the displayed equations. Underline Greek letters in red, German in green, and script in blue. The first paragraph must be capable of being used separately as a synopsis of the entire paper. In particular it should contain no bibliographic references. Please propose a heading for the odd numbered pages of less than 35 characters. Manuscripts, in triplicate, may be sent to any one of the editors. Please classify according to the scheme of Math. Reviews, Index to Vol. 39. Supply name and address of author to whom proofs should be sent. All other communications should be addressed to the managing editor, or Elaine Barth, University of California, Los Angeles, California 90024-1555-05.

There are page-charges associated with articles appearing in the Pacific Journal of Mathematics. These charges are expected to be paid by the author's University, Government Agency or Company. If the author or authors do not have access to such Institutional support these charges are waived. Single authors will receive 50 free reprints; joint authors will receive a total of 100 free reprints. Additional copies may be obtained at cost in multiples of 50 .

The Pacific Journal of Mathematics is issued monthly as of January 1966. Regular subscription rate: $\$ 190.00$ a year (6 Vols., 12 issues). Special rate: $\$ 95.00$ a year to individual members of supporting institutions.

Subscriptions, orders for numbers issued in the last three calendar years, and changes of address should be sent to Pacific Journal of Mathematics, P.O. Box 969, Carmel Valley, CA 93924, U.S.A. Old back numbers obtainable from Kraus Periodicals Co., Route 100, Millwood, NY 10546.

The Pacific Journal of Mathematics at P.O. Box 969, Carmel Valley, CA 93924 (ISSN 0030-8730) publishes 6 volumes per year. Second-class postage paid at Carmel Valley, California 93924, and additional mailing offices. Postmaster: send address changes to Pacific Journal of Mathematics, P.O. Box 969, Carmel Valley, CA 93924.

\section{PUBLISHED BY PACIFIC JOURNAL OF MATHEMATICS, A NON-PROFIT CORPORATION} Copyright (C) 1990 by Pacific Journal of Mathematics 


\section{Pacific Journal of Mathematics}

Vol. 142, No. $2 \quad$ February, 1990

Christopher J. Bishop, Bounded functions in the little Bloch space . . . . . 209

Lutz Bungart, Piecewise smooth approximations to $q$-plurisubharmonic

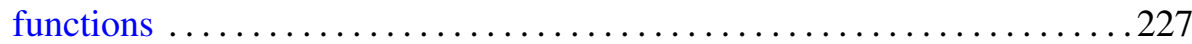

Donald John Charles Bures and Hong Sheng Yin, Outer conjugacy of

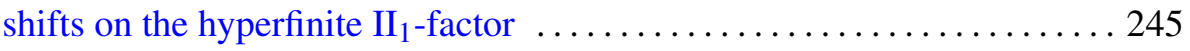

A. D. Raza Choudary, On the resultant hypersurface $\ldots \ldots \ldots \ldots \ldots 259$

Luis A. Cordero and Robert Wolak, Examples of foliations with foliated

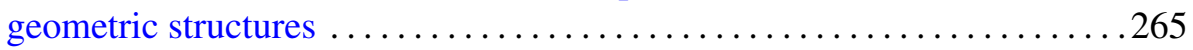

Peter J. Holden, Extension theorems for functions of vanishing mean

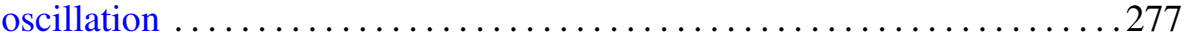

Detlef Müller, A geometric bound for maximal functions associated to convex bodies ........................................ 297

John R. Schulenberger, Time-harmonic solutions of some dissipative problems for Maxwell's equations in a three-dimensional half space . . . 313

Mark Andrew Smith and Barry Turett, Normal structure in Bochner

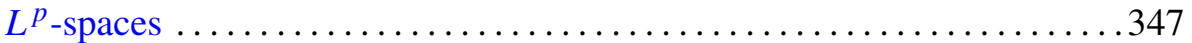

Jun-ichi Tanaka, Blaschke cocycles and generators ................... 357

R. Z. Yeh, Hyperholomorphic functions and higher order partial differential equations in the plane ................................ 379 